\title{
A REVIEW OF TELOCYTES IN CARDIOVASCULAR TISSUE AND THEIR ROLE IN ANGIOGENESIS
}

\author{
Ibsen Ongidi, Fadhila Abdulsalaam, Harry Otieno, Noel Odero, Anne Pulei, Moses Obimbo, Kevin \\ Ongeti
}

Correspondence to Ibsen H. Ongidi; Department of Human Anatomy, University of Nairobi. Email: ongidi409@gmail.com

\begin{abstract}
Telocytes are interstitial cells characterized by small cell bodies with very long and slender processes extending from them. They are present in most tissues and are most commonly found in close association with nerves, smooth muscle and microvascular networks in tissue interstitial space. Telocytes maintain tissue homeostasis in various ways including modulation of electrical activity, regulation of stem cell proliferation and angiogenesis. The role of telocytes in blood vessels stems from a structural and functional coupling to vascular endothelial and smooth muscle cells. This review explores the distribution of telocytes in cardiovascular tissues, their mechanisms in angiogenesis and application of this property in tissue regeneration. The literature search was conducted on PubMedтм, Science directтм and African Journals Online databases as well as Google Scholartm search engines. The keywords used in the literature search included 'telocytes', 'cardiovascular telocytes', 'blood vessel', 'cardiac tissue', '(neo)angiogenesis' and 'tissue regeneration'. Articles which contained the keywords and relevant citations from their reference lists were included in the study. In conclusion, telocytes display close structural relationship with cardiovascular tissues. They play an angiogenesis inducing role which may be explored as a therapeutic target in tissue repair and regeneration.
\end{abstract}

KEYWORDS : cardiovascular telocytes, interstitial Cajal-like cell, regeneration, angiogenesis

\section{INTRODUCTION}

Telocytes are a specialized type of interstitial cells characterized in electron microscopy by the presence of telopodes, which are long slender processes that extend from their cell bodies. The term 'Telocytes' was first suggested by Popescu et al. (2010) to refer to all interstitial cells of Cajal-Like Cells found in other tissues other than the gastrointestinal tract. Telocytes are ubiquitously distributed in tissues around the body and are commonly found in close contact with capillaries and other microvessels supplying skeletal muscle (Marini et al., 2018), skin (Ceafalan et al., 2012), uterus (Aleksandrovych et al., 2016), lung (Zheng et al., 2014a), esophagus (Chen et al., 2013), eyes (Luesma et al., 2013), placenta (Suciu et al., 2010) and duodenum (Cantarero et al., 2011). In the cardiovascular system, telocytes are found in the myocardium of the heart (Gherghiceanu and Popescu, 2012) and in the walls of larger arteries and veins ( $\mathrm{Li}$ et al., 2014).

Telocytes display heterocellular connections with vascular endothelial cells, smooth muscle cells and Schwann cells (Luesma et al., 2013). These include point contacts, nanocontacts and planar contacts. These contacts are atypical intercellular junctions characterized by reduced intermembrane distance but lacking structural proteins associated with classical junctions (Gherghiceanu and Popescu, 2012). The role of telocytes in tissue regeneration and 
angiogenesis has been suggested (Bei et al., 2015). The telocytes located within the extracellular matrix in close connection with blood vessels produce angiogenic factors such as vascular endothelial growth factor (VEGF) and epidermal growth factor (EGF) (Zheng et al., 2014a). These cells also demonstrate the presence of measurable quantities of proangiogenic microRNAs that influence vascular development (Manole et al., 2011). These factors are thought to cause angiogenesis as is seen in cardiac tissue treated with telocyte exosomes following vascular injury (Yang et al., 2017).
Knowledge of the role and mechanisms of telocytes in angiogenesis may influence the development of therapeutic strategies for ischemic diseases such as myocardial infarction, stroke, peripheral artery diseases and coronary heart diseases. This paper provides a summary of the structural properties of telocytes in the cardiovascular system, their mechanisms in induction of angiogenesis and value of this properrty in tissue regeneration.

\section{METHODOLOGY}

The literature search was conducted on Google Scholartm search engines and on PubMedrm, Science directrm and African Journals Online databases. The keywords used in the literature search were 'telocytes', 'cardiovascular telocytes', 'blood vessel', 'cardiac tissue', '(neo-)angiogenesis' and 'tissue regeneration'. Articles which contained the keywords and information relevant to the review were included in the study. Any additional papers from the

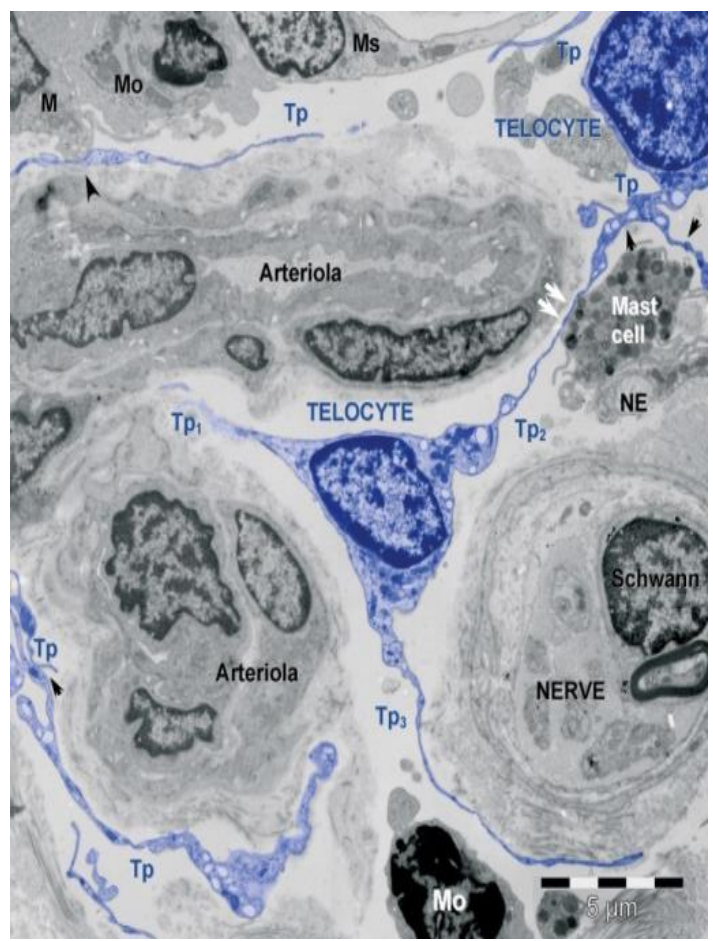

reference lists of these articles which were noted to be relevant were also included. Articles were excluded if they did not focus on telocytes and their role in angiogenesis, were not accessible for free or not available in English. The search carried out on the databases mentioned along with relevant papers identified in their reference lists yielded a total of 40 papers relevant to the review which were then reviewed.
Figure 1: Photomicrograph showing the structure of telocytes in transmission electron microscopy with digitally enhanced color (blue). The telopodes are easily noticeable (white arrows). (Adapted from Ceefalan et al., 2012). 
Anatomy Journal of Africa. 2020. Vol 9 (2): 1807 - 1815.

\section{DISCUSSION}

\section{Properties of telocytes in cardiovascular system}

Telocytes are closely related to the tissues of the cardiovascular system, including the heart and blood vessels. These cells are present in blood and in walls of large arteries and veins. They are located in the tunica adventitia of coronary arteries, internal thoracic arteries and carotid arteries (Li et al., 2014). In the walls of veins, they are located in the sub-endothelial layer (Zhang, 2016). Telocytes are also located in close contact with arterioles and capillaries supplying tissues. Investigations using transmission electron microscopy show that telocytes have two distinguishing features: 2-5 cell body prolongations referred to as telopodes and presence of distinct caveolae (Gherghiceanu and Popescu, 2012, 2005). Telopodes display a moniliform aspect characterized by alternating thin and dilated segments referred to as 'podomers' and 'podoms' respectively (Ibba-Manneschi et al., 2016) [Figure 1]. The dilated regions accommodate mitochondria, endoplasmic reticulum and caveolae. Telocytes can have varying shapes depending on the number of their telopodes: piriform for cells with one telopodes, spindle for those with two telopodes, triangular for those with three or stellate for multiple telopodes (Enciu and Popescu, 2015).

Telocytes display direct connections to other cell types such as vascular endothelial cells, vascular smooth muscle cells and cardiac myocytes (Aleksandrovych et al., 2017). These connections, located in their telopodes, allow them to communicate even with distant cells. The junctions include point contacts, nanocontacts and planar contacts (Gherghiceanu and Popescu, 2012). These junctions are not similar to classical intercellular junctions. This is because they lack electron-dense structures present on the plasma membrane or cytoplasm adjacent to the contacts that may give inference to one of the known classes of intercellular junctions. The basal lamina of both vascular endothelial cells and pericytes are diminished at the level of their heterocellular junctions with telocytes. Areas of contact between telocytes and vascular cells may act as points of communication or functional coupling (Suciu et al., 2010). This direct contact allows telocytes to influence the proliferation of vascular endothelial and smooth muscle cells. In addition, they create a scaffolding for support of stem cells that migrate to form new vascular cells during angiogenesis (Aleksandrovych et al., 2017).

\section{Role of telocytes in angiogenesis in- vivo and in-vitro}

\section{i) Evidence of the role of telocytes in angiogenesis in tissue microvascular network}

Telocytes play a role in cardiac regeneration by promoting angiogenesis, as evidenced by results from experimental research in animals. Transplantation of cardiac telocytes has been shown to increase vascular density in the myocardium of rats with induced myocardial infarction (MI), indicating an angiogenesis inducing role (Zhao et al., 2014). Telocytes may support this process angiogenesis by providing a structural and functional niche for endothelial cells. Reports have indicated that the number of telocytes increases in neo-angiogenesis induced by agents such as phytochemicals in cardiac tissue affected by MI (Nour et al., 2017).

Li et al. (2016) in a previous study demonstrated that the number of telocytes significantly increases in vascular walls after carotid artery balloon injury in rats. 
Attempts at regeneration of the injured tissue showed that the proportion of vascular smooth muscle cells in the blood vessels increased significantly when incubated with supernatant of telocytes ( $\mathrm{Li}$ et al., 2016). This suggests that these cells induce proliferation of vascular smooth muscle cells and, therefore, play a role in the repair of ischemic injury to the heart.

Experimental research on pulmonary tissue illustrates that telocytes are involved in the process of angiogenesis in the microvascular network. Expression of VEGF and EGF in human lung tissue is significantly higher when co-cultured with telocytes (Zheng et al., 2014). Telocytes also promote the proliferation of human pulmonary microvascular endothelial cells. They have been shown to cause partial recovery in human pulmonary microvascular endothelial cells injured by lipopolysaccharide (LPS) endotoxin (Zheng et al., 2014a). An article by Ma et al. (2018) correspondingly indicates that telocytes improve the proliferation of pulmonary vascular endothelial cells and cause an improvement in LPS induced inhibition of endothelial cell proliferation. Expression of VEGF and angiogenic microRNAs is reported to increase in the medium containing LPS, with a concomitant increase in the number of telocytes.

In the placenta, VEGF produced by telocytes acts as an angiogenic agent in promoting endothelial cell proliferation and vascular permeability. It also maintains newly formed blood vessels in the placenta (Suciu et al., 2010). There is a low expression of inducible nitric oxide synthase in telocytes from normal placentas. This implies a role of telocytes in the regulation of placental blood flow through an inhibitory signalling pathway that involves nitric oxide (Nizyaeva et al., 2018).

In skeletal muscle, telocytes may be crucial in the regulation of blood vessels, blood flow and muscle regeneration (Popescu et al., 2011). The role of telocytes in angiogenesis in skeletal muscle may start as early as myogenesis (Marini et al., 2018). These cells play a role in inducing angiogenesis to support myotube maturation and muscle tissue differentiation. They support skeletal muscle fibre regeneration in postnatal life by expressing VEGF for recruitment of small vessels and providing scaffolding guidance for satellite cells (Bojin et al., 2011; Popescu et al., 2011).

Telocytes in the oesophagus produce measurable amounts of VEGF and EGF (Chen et al. 2013). Secretion of proangiogenic factors by telocytes and their close spatial relations with vascular endothelial and smooth muscle cells implies their involvement in angiogenesis. Likewise, telocytes influence proliferation and regulation of blood vessels in the skin (Manole and Simionescu, 2016), female reproductive tract (Aleksandrovych et al., 2016), parotid gland (Nicolescu et al., 2012) and synovium (Rosa et al., 2018). Further studies may however be necessary to establish the role of these cells in tissue microvascular network in varying physiological and pathological states.

\section{ii) Mechanisms of telocyte induced angiogenesis}

Telocytes communicate indirectly with vascular endothelial cells and other cells of cardiovascular tissues through paracrine signalling. This is achieved by secreting microvesicles that contain factors such as VEGF which regulates vascular proliferation (Zheng and Wang, 2016). Cardiac telocytes are shown in cultures to release three types of extracellular vesicles; multivesicular cargos (tightly packed endomembrane bound vesicles), exosomes and ectosomes (Fertig et al., 2014). These vesicles contain secretory products from telocytes, including pro-angiogenic factors and microRNAs. 
Cardiac telocytes increase their expression of VEGF and the inflammatory protein interleukin-6 (IL-6) when co-cultured with cardiac stem cells in vitro (Albulescu et al., 2015). The mechanisms of telocyte induced angiogenesis in the heart include the expression of stem cell markers and VEGF. These are important factors which promote vascular smooth muscle and endothelial cell development from angioblast stem cells (Albulescu et al., 2015).

VEGF and their receptors are key signalling factors that initiate the process of angiogenesis (Karamysheva, 2018). The production of such angiogenic factors by telocytes indicate their important role in the development of blood vessels. Telocytes also expresses nitric oxide which is an important molecule in the development and organization of new blood vessels (Aleksandrovych et al., 2016). From these studies, it can be proposed that telocytes participate in angiogenesis, both in physiological and pathological conditions. Telocytes induce angiogenesis by the release of VEGF, microRNAs and other angiogenesis promoting factors. These increase angioblast differentiation into vascular cells and provides a scaffold for the migration of these developing cells.

\section{Role of telocytes in vascular diseases}

Existence of hypoxia and oxidative stress in the placenta in women with preeclampsia leads to a reduction in the number of telocytes and impairment of their function (Bosco et al., 2015). This is evidenced by morphological changes in telocytes including thickening of telopodes and decreased the content of endoplasmic reticulum (Nizyaeva et al., 2018). Hypoxia and oxidative stress may be one of the causes of vascular impairment in the placenta during pre-eclampsia. Telocytes have been revealed not only promote angiogenesis but also play a role in placental blood flow regulation through nitric oxide signalling (Suciu et al., 2010). Further exploration of this relationship between telocytes and the placental microvascular network can improve the understanding of the pathology of preeclampsia and intrauterine growth restriction.

In the female reproductive tract, the role of telocytes can predict a crucial role in the development of uterine leiomyomas, endometriosis, salpingitis and cancers (Aleksandrovych et al., 2016). Loss of uterine telocytes in uterine leiomyoma is postulated to be responsible for the decreased blood vessel formation observed (Varga et al., 2018). This may cause a shift to anaerobic metabolism in smooth muscle cells, a factor that favours the development of leiomyoma. This is consistent with the reporting of decreased angiogenesis and vascular density by Sajewicz et al. (2016) and Uluer et al., (2015) respectively in studies investigating uterine leiomyoma pathology.

Neo-angiogenesis is partially responsible for some of the clinical signs seen in psoriasis, a common skin condition. Evidence of a reduction in telocyte density around blood vessels in skin affected by psoriasis suggests that they may play a role in the development of the disease (Manole et al., 2015). Telocyte expression in perivascular tissue, however, improves with the application of corticosteroids on the affected skin. Phenotypic changes in vascular smooth muscle cells not surrounded by telocytes in the papillary dermis of psoriatic skin partly explains the impairment in blood vessel structure in the disease (Manole et al., 2015).

The role of telocytes in other diseases that stem from impaired angiogenesis remains to be established. Their role in tumour stroma formation has been hypothesized to be due to the secretion of angiogenic inducers, among other factors (Diaz-Flores 
et al., 2016). Rosa et al. (2017) also proposed that telocytes can be players in the development of degenerative joint diseases such as arthritis, lupus and gout. These are diseases that exhibit synovial inflammation and blood vessel proliferation as predominant features.

\section{Therapeutic application for angiogenic role of telocytes}

Telocytes are not yet used therapeutically for vascular injury. However, several studies have attempted to establish their benefit in animal experimental models for treating myocardial infarction. For example, transplantation of cardiac telocytes causes increased angiogenesis and reduces the infarct size in rat experimental models of myocardial infarction (Zhao et al., 2014). This is associated with an overall enhancement of myocardial function as shown by higher left ventricle ejection fraction. The tissue regeneration due to augmented angiogenesis caused by transplanted telocytes also occurs without excessive fibrosis in heart muscle. Telocytes can be utilized to develop novel therapeutic targets in tissue engineering and regeneration (Aleksandrovych et al., 2017). Their angiogenic role in various tissues presumably provides a regenerative ability which may be used alone or in combination with stem cell therapy for treating MI (Boos et al., 2016).

Telocyte therapy can similarly be developed in clinical trials for the treatment of lung diseases. In vitro experiments have shown that telocyte administration causes an improvement of function in human pulmonary endothelial cells injured by LPS (Ma et al., 2018; Zheng et al., 2014a). Evidence suggests there is presence of telocytes in the limbus and uvea of the eye (Luesma et al., 2013). The potential of these cells in neo-angiogenesis can be explored to generate therapy in neovascular eye diseases such as age-related macular degeneration (Bei et al., 2015; Campochiaro, 2013).

Formation of mature, well organized and stable vasculature is a key goal in tissue engineering, regenerative medicine, therapeutic angiogenesis and treatment of vascular disease (Suciu et al., 2012). Regenerative therapy using stem cells alone may fail in some cases due to inadequate formation of a blood vessel scaffold in the newly formed tissues (Pellegrini and Popescu, 2011). Stem cells, when coadministered with telocytes, would provide better results for such therapy than when administered alone (Popescu et al., 2015). This is explained by their ability to induce angiogenesis.

The physiological capacity of telocytes to induce the formation of stable vasculature may be valuable and remains to be fully explored in clinical trials. The role of telocytes in supporting angiogenesis as shown in animal experiments should be tailored to create therapies for the regeneration of human tissues (Kucybala et al., 2017). These therapies may be used to treat diseases in tissues which naturally have a low regenerative capacity through genetic engineering of telocytes (Boos et al., 2016).

\section{CONCLUSION}

Telocytes have a unique relationship with vascular endothelial cells in tissue microvasculature as well as endothelial and smooth muscle cells in larger vessels. They are mechanically coupled with these cells through contacts that exist at telopodes. They also exhibit paracrine communication with these cells via secretion of microRNAs, nitric oxide and vascular growth factors such as VEGF and EGF which are key signalling factors in the process of angiogenesis. These cells are linked to the pathogenesis of ischemic diseases and further experiments may provide new 
Anatomy Journal of Africa. 2020. Vol 9 (2): 1807 - 1815.

strategies in their treatment. The angiogenesis inducing role of telocytes may be explored as a therapeutic target in tissue repair and regeneration e.g. by direct transplantation of genetically engineered telocytes.

\section{REFERENCES}

1. Albulescu R, Tanase C, Codrici E, Popescu DI, Cretoiu SM, Popescu LM. 2015. The secretome of myocardial telocytes modulates the activity of cardiac stem cells. J Cell Mol Med, 19(8): 1783-1794.

2. Aleksandrovych V, Pasternak A, Basta P, Sajewicz M, Walocha JA, Gil K. 2017. Telocytes: facts, speculations and myths. Folia Med Cracov, 57(1): 5-22.

3. Aleksandrovych V, Walocha JA, Gil K. 2016. Telocytes in female reproductive system (human and animal). J Cell Mol Med, 20(6): 994-1000.

4. Bei Y, Wang F, Yang C, Xiao J. 2015. Telocytes in regenerative medicine. J Cell Mol Med 19(7): 1441-1454.

5. Bojin FM, Gavriliuc OI, Cristea MI, Tanasie G, Tatu CS, Panaitescu C, Paunescu V. 2011. Telocytes within human skeletal muscle stem cell niche. J Cell Mol Med, 15(10): 2269-2272

6. Boos AM, Weigand A, Brodbeck R, Beier JP, Arkudas A, Horch RE. 2016. The potential role of telocytes in tissue engineering and regenerative medicine. Seminars in cell \& developmental biology; Vol 55, pp 70-78; Academic Press.

7. Bosco C, Díaz E, Gutiérrez R, González J, Parra-Cordero M, Rodrigo R, Barja P. 2015. A putative role for telocytes in placental barrier impairment during preeclampsia. Med Hypotheses, 84(1): 72-77.

8. Campochiaro PA. 2013. Ocular neovascularization. J Mol Med, 91(3): 311-321.

9. Ceafalan L, Gherghiceanu M, Popescu LM, Simionescu O. 2012. Telocytes in human skin-are they involved in skin regeneration? J Cell Mol Med, 16(7): 1405-1420

10. Chen X, Zheng Y, Manole CG, Wang X, Wang Q. 2013. Telocytes in human oesophagus. J Cell Mol Med, 17(11): 506-1512.

11. Díaz-Flores L, Gutiérrez R, Diaz-Flores Jr L, Goméz MG, Saez FJ, Madrid JF. 2016. Behavior of telocytes during physiopathological activation. Seminars in cell \& developmental biology; Vol 55, pp 50-61; Academic Press.

12. Enciu AM, Popescu LM. 2015. Telopodes of telocytes are influenced in vitro by redox conditions and ageing. Mol Cell Biochem, 410(1-2): 165-174.

13. Fertig ET, Gherghiceanu M, Popescu LM. 2014. Extracellular vesicles release by cardiac telocytes: electron microscopy and electron tomography. J Cell Mol Med, 18(10): 1938-1943.

14. Gherghiceanu M, Popescu LM. 2012 Cardiac telocytes-their junctions and functional implications. Cell Tissue Res, 348(2): 265-279.

15. Karamysheva AF. 2008. Mechanisms of angiogenesis. Biochemistry (Moscow), 73(7): 751.

16. Kucybala I, Janas P, Ciuk S, Cholopiak W, Klimek-Piotrowska W, Holda MK. 2017 A comprehensive guide to telocytes and their great potential in cardiovascular system. Bratisl Med J, 118(5): 302-309.

17. Li H, Lu S, Liu H, Ge J, Zhang H. 2014. Scanning electron microscope evidence of telocytes in vasculature. J Cell Mol Med, 18(7): 1486-1489. 
18. Li Y, Zhang X, Gao J, Xiao H, Xu M. 2016. Increased telocytes involved in the proliferation of vascular smooth muscle cells in rat carotid artery balloon injury. Sci China Life Sci, 59(7): 678-685.

19. Luesma MJ, Gherghiceanu M, Popescu LM. 2013. Telocytes and stem cells in limbus and uvea of mouse eye. J Cell Mol Med, 17(8): 1016-1024.

20. Ma R, Wu P, Shi Q, Song D, Fang H. 2018. Telocytes promote VEGF expression and alleviate ventilator-induced lung injury in mice. Acta Biochim Biophys Sin (Shanghai), 50(8): 817-825.

21. Manole CG, Simionescu O. 2016. The Cutaneous Telocytes. In: Wang Xiangdong, Cretoiu Dragos (Eds.) Telocytes. Springer, Singapore.

22. Manole CG, Cismaşiu V, Gherghiceanu M, Popescu LM. 2011. Experimental acute myocardial infarction: telocytes involvement in neo-angiogenesis. J Cell Mol Med, 15(11): 2284-2296.

23. Manole CG, Gherghiceanu M, Simionescu O. 2015. Telocyte dynamics in psoriasis. J Cell Mol Med, 19(7): 1504-1519.

24. Marini M, Manetti M, Rosa I, Ibba-Manneschi L, Sgambati E. 2018. Telocytes in human fetal skeletal muscle interstitium during early myogenesis. Acta Histochem, 120(5): 397-404.

25. Nicolescu MI, Bucur A, Dinca O, Rusu MC, Popescu LM. 2012. Telocytes in parotid glands. Anat Rec (Hoboken), 295(3): 378-385.

26. Nizyaeva NV, Sukhacheva TV, Serov RA, Kulikova GV, Nagovitsyna MN, Kan NE, Tyutyunnik VL, Pavlovich SV, Poltavtseva RA, Yarotskaya EL, Hchegolev AI. 2018. Ultrastructural and Immunohistochemical Features of Telocytes in Placental Villi in Preeclampsia. Sci Rep, 8(1): 3453.

27. Nour MS, Sarhan NR, Mazroa SA, Gawish SA. 2017. Histological and immunohistochemical study of cardiac telocytes in a rat model of isoproterenolinduced myocardial infarction with a reference to the effect of grape seed extract. Acta Histochem, 119(7): 747-758.

28. Pellegrini MSF, Popescu LM . 2011. Telocytes. Biomol Concepts, 2(6): 481-489.

29. Popescu LM, Curici A, Wang E, Zhang H, Hu S, Gherghiceanu M. 2015. Telocytes and putative stem cells in ageing human heart. J Cell Mol Med, 19(1): 31-45.

30. Popescu LM, Manole E, Şerboiu CS, Manole CG, Suciu LC, Gherghiceanu M, Popescu BO. 2011. Identification of telocytes in skeletal muscle interstitium: implication for muscle regeneration. J Cell Mol Med, 15(6): 1379-1392.

31. Rosa I, Marini M, Guasti D, Ibba-Manneschi L, Manetti M. 2018. Morphological evidence of telocytes in human synovium. Sci Rep, 8(1): 3581.

32. Sajewicz M, Konarska M, Wrona AN, Aleksandrovych V, Bereza T, Komnata K, Solewski B, Maleszka A, Depukat P, Warchol L. 2016. Vascular density, angiogenesis and pro-angiogenic factors in uterine fibroids. Folia Med Cracov, 56(1): 27-32.

33. Suciu L, Popescu LM, Gherghiceanu M, Regalia T, Nicolescu MI, Hinescu ME, Faussone-Pellegrini MS (2010) Telocytes in human term placenta: morphology and phenotype. Cells Tissues Organs, 192(5): 325-339.

34. Suciu LC, Popescu BO, Kostin S, Popescu LM. 2012. Platelet-derived growth factor receptor- $\beta$-positive telocytes in skeletal muscle interstitium. J Cell Mol Med, 16(4): 701-707.

35. Uluer ET, Inan S, Ozbilgin K, Karaca F, Dicle N, Sancı M. 2015. The role of hypoxiarelated angiogenesis in uterine smooth muscle tumors. Biotech Histochem, 90(2): 102-110. 
36. Varga I, Klein M, Urban L, Danihel Jr L, Polak S, Daniel Sr L (2018) Recently discovered interstitial cells "telocytes" as players in the pathogenesis of uterine leiomyomas. Med Hypotheses, 110: 64-67.

37. Zhang H. 2016. Vascular Telocytes. In: Wang Xiangdong, Cretoiu Dragos (Eds.) Telocytes. Springer, Singapore.

38. Zhao B, Liao Z, Chen S, Yuan Z, Yilin C, Lee KK, Qi X, Shen X, Zheng X, Quinn T, Cai D. 2014. Intramyocardial transplantation of cardiac telocytes decreases myocardial infarction and improves post-infarcted cardiac function in rats. J Cell Mol Med, 18(5): 780-789.

39. Zheng Y, Wang X. 2016. Roles of Telocytes in the Development of Angiogenesis. In: Wang Xiangdong, Cretoiu Dragos (Eds.) Telocytes. Springer, Singapore.

40. Zheng Y, Chen X, Qian M, Zhang M, Zhang D, Bai C, Wang Q, Wang X. 2014. Human lung telocytes could promote the proliferation and angiogenesis of human pulmonary microvascular endothelial cells in vitro. Mol Cell Ther, 2(1): 3. 